\title{
MULTIPLIERS FOR THE SYMMETRY GROUPS OF P-ADIC SPACETIME
}

\author{
V. S. VARADARAJAN \\ Department of Mathematics \\ University of California, Los Angeles, CA 90095-1555, USA. \\ vsv@math.ucla.edu
}

\begin{abstract}
The consequences for particle classification of the Volovich hypothesis that spacetime geometry is non-archimedean at the Planck scale are explored. The multiplier groups and universal topological central extensions of the $p$-adic Poincaré and Galilean groups are determined.
\end{abstract}

Key words: Volovich hypothesis, multipliers, Poicaré group, Galilean group

Mathematics subject classification 2000: 22E35, 22E41, 22E50

\section{The Volovich hypothesis.}

In attempting to unify gravitation and quantum theory people have encountered deep obstacles connected with the structure of spacetime at very short distances and times. The idea of extended particles (strings, branes) makes sense in this context. Already in the early 1970's Beltrametti and his collaborators [1] [2] [3] had examined the possibility that spacetime geometry may be based on a $p$-adic or even a finite field. In important work [4] [5] in 1987 Volovich made the bold hypothesis that spacetime geometry may be non archimedean at the Planck scale. The basis for this suggestion is the idea that principles of general relativity and quantum theory forbid any measurement of distances less than the Planck length, so that the archimedean 
axiom might cease to have validity in sub-Planckian regions. In this paper we examine the consequences of this hypothesis for particle classification. Since elementary particles define PUIR's (=projective unitary irreducible representations) of the spacetime symmetry group, as a first step in this program it becomes necessary to determine all the phase factors (=multipliers) that enter the PUIR's. We obtain a generalization for the $p$-adic Poincaré and Galilean groups of the famous theorem of E. Wigner [6] that all PUR 's of the real Poincaré group become UR's when lifted to its two-fold cover.

\section{Multipliers.}

We begin with a very brief summary of the theory of multipliers (for more details see [7]). Let $G$ be any lesc (=locally compact second countable) group. A PUR (=projective unitary representation) of $G$ is a Borel map $U$ of $G$ into the unitary group of a complex separable Hilbert space $\mathcal{H}=\mathcal{H}(U)$ such that $U(1)=1$ and $U(x) U(y)=m(x, y) U(x y)$ for all $x, y \in G$. We call $m$ the multiplier for $U$, and $U$ an $m$-representation; $m$ is Borel, $m(x, y) \in T$ where $T$ is the group of complex numbers of absolute value 1 , and satisfies the relations

$$
m(x, 1)=m(1, y)=1, \quad m(x y, z) m(x, y)=m(x, y z) m(y, z) .
$$

Any Borel function $m(G \times G \longrightarrow T)$ satisfying the above relations is called a multiplier for $G$. If $m$ is a multiplier there is always an $m$-representation, even an irreducible one ([7], p. 249 and p. 259). Multipliers $m, m^{\prime}$ are equivalent $\left(m \simeq m^{\prime}\right)$ if $m^{\prime}(x, y)=m(x, y) a(x y) a(x)^{-1} a(y)^{-1}$ for some Borel map $a(G \longrightarrow T)$ with $a(1)=1$. The set of multipliers under pointwise multiplication is an abelian group $Z(G)$ and the trivial ones, i.e., those $\simeq 1$ form a subgroup $B(G) ; H^{2}(G):=Z(G) / B(G)$ is the multiplier group of $G$.

Given $m \in Z(G)$ one can build a central extension $E_{m}$ of $G$ by $T$,

$$
1 \longrightarrow T \longrightarrow E_{m} \longrightarrow G \longrightarrow 1
$$

where $E_{m}=G \times T$ with the multiplication $(x, t)\left(x^{\prime}, t^{\prime}\right)=\left(x x^{\prime}, t t^{\prime} m\left(x, x^{\prime}\right)\right)$ and maps $t \mapsto(1, t),(x, t) \mapsto x$. With its so-called Mackey-Weil topology, $E_{m}$ becomes lcsc, the maps above are continuous, and $E_{m}$ is a topological central extension (t.c.e.) of $G$; moreover, any $m$-representation lifts to a UR of $E_{m}$ which restricts to $(1, \mathrm{t})$ as $t 1$, and this lifting gives a bijection between $m$ representations of $G$ and such UR's of $E_{m}$. If $m$ is continuous, the topology 
on $E_{m}$ is the product topology. The extension $E_{m}$ varies with $m$ and so it is natural to ask if there is a universal one(u.t.c.e). A t.c.e

$$
1 \longrightarrow \mathbf{A} \longrightarrow \mathbf{E} \longrightarrow G \longrightarrow 1
$$

is called universal if every t.c.e $E$ is an image of $\mathbf{E}$ by a unique map $\varphi(\mathbf{E} \longrightarrow$ $E$ ). A given t.c.e $E$ of $G$ is a u.t.c.e if and only if (a) the commutator subgroup $(E, E)$ is dense in $E$ and (b) $H^{2}(E)=0$. In this case we write $E=G^{\sim}$. Any PUR of $G$ then lifts uniquely to an ordinary UR of $G^{\sim}$. Thus $G^{\sim}$, which is unique up to unique isomorphism, may be viewed as the effective symmetry group of the system. Note that for $G$ to have a t.c.e. it is necessary that $(G, G)$ be dense in $G$.

We have used Borel cocycles to define the multiplier group. For totally disconnected groups life is much simpler, as was shown by D. Wigner [8] who used the following special case of a selection theorem of E. Michael [9]: if $Y, X$ are locally compact Hausdorff spaces with $X$ t.d. and $f(Y \longrightarrow X)$ is an open continuous surjective map, we can find a continuous map $g(X \longrightarrow Y)$ such that $f \circ g$ is the identity on $X$.

Lemma 1. Let $G$ be a t.d. group. (a) Any multiplier for $G$ is equivalent to a continuous one. (b) If $m_{1} \simeq m_{2}$ where $m_{i} \in Z(G)$ are continuous, and $a(G \longrightarrow T)$ is a Borel map with $a(1)=1$ and $m_{2}(g, h)=$ $m_{1}(g, h) a(g h) a(g)^{-1} a(h)^{-1}$, then a is continuous. (c) If $m$ is a continuous multiplier for $G$ and $U$ an m-representation, then $U$ is already continuous. In particular the natural map $H_{c}^{2}(G) \longrightarrow H^{2}(G)$ is an isomorphism.

Proof. (a) Let $m \in Z(G)$ and $U$ an $m$-representation. Then $V((x, t) \longmapsto$ $t U(x))$ is a continuous UR of $E_{m}$. By the theorem of Michael we can find a continuous map $x \longmapsto(x, a(x))$ of $G$ into $E_{m}$. If $U^{\prime}(x)=V((x, a(x))=$ $a(x) U(x)$, then $U^{\prime}$ is a PUR with a multiplier $m^{\prime}$ equivalent to $m$; as $U^{\prime}$ is continuous, so is $m^{\prime}$.

(b) The map $f$ from $E_{m_{1}}$ to $E_{m_{2}}$ given by $(x, t) \longmapsto(x, t a(x))$ is a Borel homomorphism and hence continuous. Since $E_{m_{2}}$ has the product topology, the projection $P\left(E_{m_{2}} \longrightarrow T\right)$ is continuous. If $g$ is the map $x \longmapsto(x, 1)$ from $G$ to $E_{m_{1}}$, then $g$ is continuous because $E_{m_{1}}$ has the product topology. The continuity of $a$ follows from $a(x)=(P \circ f \circ g)(x)$.

(c) Since $m$ is continuous, the map $x \longmapsto(x, 1)$ from $G$ to $E_{m}$ is continuous and $U(x)=V((x, 1))$ is continuous in $x$. 
Lemma 2. Let $G$ be lcsc and $E$ a t.c.e. of $G$ with $(E, E)$ dense in $E$. If all PUR's of $G$ become unitarizable when lifted to $E$ then $E$ is the u.t.c.e. of $G$.

Proof. Let $C$ be the kernel $E \longrightarrow G$. We must prove that $H^{2}(E)=0([21])$. Let $m$ be a multiplier of $E$ and $U$ an irreducible $m$-representation. We have $h c h^{-1}=c$ for all $h \in E, c \in C$ and hence, with $c$ fixed, $U(h) U(c) U(h)^{-1}=$ $\chi(h) U(c)$ where $|\chi(h)|=1$. Now $U\left(h_{1}\right) U\left(h_{2}\right)=m\left(h_{1}, h_{2}\right) U\left(h_{1} h_{2}\right)\left(h_{1}, h_{2} \in\right.$ $E)$, from which it follows that $\chi$ is a character in $h$. As $(E, E)$ is dense in $E$ we may conclude that $\chi(h)=1$. In other words each $U(c)$ commutes with $U$ and so must be a scalar. So $U$ descends to $G$ as a PUR. But all PUR's of $G$ lift to UR's of $E$. Hence $U$ can be normalized to a UR on $E$.

The study of multipliers of $p$-adic linear groups was initiated by C. C. Moore [10] and continued by G. Prasad-M. S. Raghunathan [11] and G. Prasad-A. S. Rapinchuk in [12]. If $\mathbf{G}$ is an absolutely almost simple, simply connected $k$-isotropic linear algebraic group defined over a local field $k$, then for $G=\mathbf{G}(k), H^{2}(G)$ is isomorphic to the (finite) group $\mu(k)$ of all roots of unity in $k$; this implies that $G^{\sim}$ exists and the kernel of $G^{\sim} \longrightarrow G$ is the dual group of $\mu(k)$. If $\mathbf{G}$ is only semi simple, $G^{\sim}$ will exist and $G^{\sim} \longrightarrow G$ will have finite kernel, provided the simple factors of $\mathbf{G}$ are defined and isotropic over $\mathbf{Q}_{p}$. The hypotheses on $\mathbf{G}$ are satisfied if $\mathbf{G}=\operatorname{Spin}(\mathbf{V})$ of a quadratic vector space $\mathbf{V}$ defined and isotropic over $k$ and of dimension $\geq 3$ but $\neq 4$, with the proviso that in dimension 4 the Witt index is 2 ..

\section{Multipliers for semi direct products.}

Our entire work is based on a theorem of Mackey [13] (pp. 303-305) on multipliers for semi direct products. We have a semi direct product $H=$ $A \times^{\prime} G$ where $A, G$ are lcsc and $A$ is abelian. Let $A^{*}$ be the dual of $A$. We are interested in multipliers for $H$ which are trivial when restricted to $A$ and $G$. This requires us to introduce the cohomology group $H^{1}\left(G, A^{*}\right)$. Let $R$ be any lcsc abelian group with $G$ acting on $R$. An 1-cocycle for $G$ with coefficients in $R$ is a Borel map $f(G \longrightarrow R)$ such that $f\left(g g^{\prime}\right)=f(g)+$ $g \cdot f\left(g^{\prime}\right)\left(g, g^{\prime} \in G\right)$. This is equivalent to saying that $g \longmapsto(f(g), g)$ is a Borel homomorphism of $G$ into the semi direct product $R \times \times^{\prime} G$ [14]. So $f(1)=0$ and $f$ is automatically continuous. The abelian group of (continuous) 1cocycles is denoted by $Z^{1}(G, R)$, the coboundaries are the cocycles of the 
form $g \mapsto g \cdot r-r$ for some $r \in R$, forming the subgroup $B(G, R)$, and $Z^{1}(G, R) / B^{1}(G, R)=: H^{1}(G, R)$.

Let $M_{0}(H)$ be the group of multipliers for $H$ which are trivial when restricted to $A$ and $G$, and $M_{00}(H)$ the subgroup of those equal to 1 on $A$ and $G$. Let $\left[M_{0}(H)\right]=\left[M_{00}(H)\right]$ be the image in $H^{2}(H)$. Following an idea that goes back to E. Wigner [6] we wish to relate $\left[M_{00}(H)\right]$ with $H^{1}\left(G, A^{*}\right)$.

Theorem 1 (Mackey). We have $\left[M_{0}(H)\right] \simeq H^{1}\left(G, A^{*}\right)$. Moreover, all multipliers in $M_{00}(H)$ are equivalent to continuous ones.

Proof. Let $m \in M_{00}(H)$ and consider the section $\gamma: a g \longmapsto(a g, 1)$ of $E_{m}$. Then $\gamma(g) \gamma(a) \gamma(g)^{-1}=\gamma(g[a]) \omega_{m}(a, g)=\omega_{m}(a, g) \gamma\left(g a g^{-1}\right)$ where

$$
\omega_{m}(a, g)=\frac{m(g, a) m\left(g a, g^{-1}\right)}{m\left(g, g^{-1}\right)}=\frac{m(g, a)}{m(g[a], g)} \in T .
$$

Since $m=1$ on $A \times A, G \times G, \gamma$ is a homomorphism separately on $A$ and $G$. It follows from this (or by direct verification) that for fixed $g \in G$, the map $a \mapsto \omega_{m}(a, g)$ is a character of $A$ (recall that Borel homomorphisms of $A$ into $T$ are characters), while for fixed $a \in A$, we have $\omega_{m}\left(a, g_{1} g_{2}\right)=$ $\omega_{m}\left(a, g_{2}\right) \omega_{m}\left(g_{2}[a], g_{1}\right) \quad\left(g_{1}, g_{2} \in G\right)$. Let us write $A^{*}$ additively. If we set $\theta_{m}(g)(a)=\omega_{m}\left(a, g^{-1}\right)$, then $\theta_{m} \in Z^{1}\left(G, A^{*}\right)$ and $m \mapsto \theta_{m}$ is a morphism from $M_{00}(H)$ into $Z^{1}\left(G, A^{*}\right)$. We wish to prove that this induces an isomorphism in cohomology.

Let $m^{\prime} \in M_{00}(H), m^{\prime} \simeq m$. So, for a Borel $f(H \longrightarrow T)$ with $f(1)=1$,

$$
m^{\prime}\left(a g, a^{\prime} g^{\prime}\right)=f\left(\left(a g\left[a^{\prime}\right]\right) g g^{\prime}\right) f(a g)^{-1} f\left(a^{\prime} g^{\prime}\right)^{-1} m\left(a g, a^{\prime} g^{\prime}\right) .
$$

Note that $f$ is a character on $A$ as well as $G$. We then find

$$
\omega_{m^{\prime}}(a, g)=\frac{f(g[a])}{f(a)} \omega_{m}(a, g), \quad \theta_{m^{\prime}}(g)=\theta_{m}(g)+(g \cdot f-f) .
$$

So $\theta_{m}$ and $\theta_{m^{\prime}}$ define the same element in $H^{1}\left(G, A^{*}\right)$.

Injectivity. Let $\theta_{m}(g)=g \cdot f-f$ for some character $f \in A^{*}$. We view $f$ as a map of $A$ into $T \subset E_{m}$. Then $\omega_{m}(a, g)=f(g[a]) f(a)^{-1}$. Let $\gamma$ be the map $h \mapsto(h, 1)$ of $H$ into $E_{m}$. Write $\delta(a)=f(a) \gamma(a), \quad \delta(g)=$ $\gamma(g), \quad \delta(a g)=\delta(a) \delta(g)$ and note that $\delta$ is a homomorphism on $A$ and $G$. Then $\delta$ is a section and we shall prove that it is a homomorphism. But $\delta\left(a g . a^{\prime} g^{\prime}\right)=\delta(a) f\left(g\left[a^{\prime}\right]\right) \gamma\left(g\left[a^{\prime}\right]\right) \gamma(g) \gamma\left(g^{\prime}\right)$. Now

$$
f\left(g\left[a^{\prime}\right]\right)=f\left(a^{\prime}\right) \omega_{m}\left(a^{\prime}, g\right)=f\left(a^{\prime}\right) \gamma(g) \gamma\left(a^{\prime}\right) \gamma(g)^{-1}\left(\gamma\left(g\left[a^{\prime}\right]\right)\right)^{-1}
$$


so that $\delta\left(a g \cdot a^{\prime} g^{\prime}\right)=\delta(a g) \delta\left(a^{\prime} g^{\prime}\right)$ after a calculation. Thus $E_{m}$ is split by $\delta$.

Surjectivity. Given $\theta \in Z^{1}\left(G, A^{*}\right)$ let $\omega(a, g)=\theta\left(g^{-1}\right)(a) ; \omega$ is continuous on $A \times^{\prime} G$. If we define $m$ by $m\left(a g, a^{\prime} g^{\prime}\right)=\omega\left(a^{\prime}, g\right)$, it is straightforward to verify that $m \in M_{00}(H)$ and $\omega=\omega_{m}$. The t.c.e. defined by $m$ is constructed as follows. We define an action of $G$ on $A \times T$ (product group) by $g[(a, t)]=(g[a], t \omega(a, g))$. It is immediate from the properties of $\omega$ that this is a continuous action of $G$ on $A \times T$. Hence we can form the semi direct product $E=(A \times T) \times^{\prime} G$. It is easy to check that $E$ is a t.c.e of $H$ by $T$ and that $m$ is the multiplier defined by the section $\gamma(a g)=((a, 1), g)$. In particular $m$ is continuous so that all multipliers in $M_{00}(H)$ are equivalent to continuous ones.

Corollary 1. If $\omega$ is given and we define $m$ by

$$
m\left(a g, a^{\prime} g^{\prime}\right)=\omega\left(a^{\prime}, g\right)
$$

then $m$ is a continuous multiplier and $\omega_{m}=\omega$.

We now examine which multipliers of $A$ arise as restrictions of multipliers for $H=A \times^{\prime} G$. A bicharacter for $A$ is a continuous map $b(A \times A \longrightarrow T)$ which is a character in each argument; it is alternating if $b(x, y) b(y, x)=1$ and $b(x, x)=1$ for $x, y \in A$. All bicharacters are continuous multipliers. Let $\Lambda^{2}(A)$ be the abelian group of all alternating bicharacters for $A$. If $m$ is a multiplier for $A$ and we set $m^{\sim}(x, y)=m(x, y) m(y, x)^{-1}$, then $m^{\sim} \in \Lambda^{2}(A)$, and $m \mapsto m^{\sim}$ is a homomorphism which induces an injection of $H^{2}(A)$ into $\Lambda^{2}(A)$. Suppose now that $A$ is 2-regular, i.e., $a \mapsto 2 a$ is an isomorphism of the lcsc group $A$ onto itself. Then the map $H^{2}(A) \longrightarrow \Lambda^{2}(A)$ is an isomorphism. In this case the map that takes an element of $\Lambda^{2}(A)$ to its image in $H^{2}(A)$ is an isomorphism. Also $G$ acts on $\Lambda^{2}(A)[16]$.

Proposition 1. Let $A$ be 2-regular. If 1 is the only element of $\Lambda^{2}(A)$ invariant under $G$, then, for any multiplier $m$ for $H$, the restriction of $m$ to $A \times A$ is trivial and so $m \simeq m^{\prime}$ where $m^{\prime}=1$ on $A \times A$. If $m$ is continuous, we can choose $m^{\prime}$ to be continuous also.

Proof. Let $U$ be an $m$-representation for $H$ and let $m_{A}=\left.m\right|_{A \times A}$. Then, for fixed $g, U(g) U(a) U(g)^{-1}=t(a) U(g[a])$ where $t(a) \in T$. Now $a \mapsto$ $U(g) U(a) U(a)^{-1}$ is an $m_{A}$-repesentation of $A$, while $a \mapsto t(a) U(g[a])$ is an $m_{A}^{\prime}$-representation of $A$ where $m_{A}^{\prime}(a, b)=m(g[a], g[b]) t(a) t(b) t(a b)^{-1}$. Thus 
$m_{A}=m_{A}^{\prime}$ showing that $m_{A} \simeq\left(m_{A}\right)_{g}$ where $\left(m_{A}\right)_{g}(a, b)=m_{A}(g[a], g[b])$. This means that $m_{\tilde{A}}^{\tilde{}}=\left(m_{A}\right)_{g}^{\tilde{g}}=\left(m_{\tilde{A}}^{\tilde{}}\right)^{g}$ in $\Lambda^{2}(A)$. So $m_{\tilde{A}}$ is $G$-invariant, hence must be 1 . If $m$ is continuous, we use [16] (p. 535, proof of lemma 2) to find a continuous $f(H \longrightarrow T)$ such that $m_{A}(a, b)=f(a) f(b) f(a b)^{-1}(a, b \in$ $A)$. Define $m^{\prime}(x, y)=m(x, y) f(x)^{-1} f(y)^{-1} f(x y)$.

\section{Multipliers for $p$-adic vector spaces.}

We consider a finite dimensional vector space $V$ over $\mathbf{Q}_{p}$, the field of $p$-adic numbers, and a $p$-adic Lie group $G$ acting on $V$ (see [17] for the basics of $p$-adic manifolds and groups). From now on we fix a non trivial additive character $\psi: \mathbf{Q}_{p} \longrightarrow T$. It is known that the kernel of $\psi$ is a compact open subgroup of $\mathbf{Q}_{p}$.

Lemma 3. Any additive continuous map between finite dimensional $\mathbf{Q}_{p^{-}}$ vector spaces is $\mathbf{Q}_{p}$-linear.

Proof. Let $F\left(U_{1} \longrightarrow U_{2}\right)$ be additive and continuous. If $u \in U_{1}$, we have $F(m u)=m F(u)$ for integers $m$, and $m F(u)=F(m u)=F(n(m / n) u)=$ $n F(m / n) u)$ so that $F(q u)=q F(u)$ for all rational $q$. By continuity $F(c u)=$ $c F(u)$ for all $c \in \mathbf{Q}_{p}$.

Let $C_{n}=C_{n}(V)$ be the abelian group of $n$-characters of $V$, namely, the continuous maps $V^{n} \longrightarrow T$ which are characters in each argument topologized by uniform convergence on compacts. Let $M_{n}$ be the vector space of $n$-linear maps of $V^{n}$ into $\mathbf{Q}_{p}$. Then $\beta \mapsto \psi(\beta)$ is a continuous homomorphism of $M_{n}$ into $C_{n}$. This map is one-one. If $\psi\left(h\left(x_{1}, \ldots, x_{n}\right)\right)=1$ for all $\left(x_{i}\right)$, then $\left|h\left(x_{1}, \ldots, x_{n}\right)\right|_{p} \leq p^{r}$ for some fixed $r$ and all $\left(x_{i}\right) \in V^{n}$, showing that $h\left(x_{1}, \ldots, x_{n}\right)=0$.

Proposition 2. The map $f \mapsto \psi(f)$ is a topological additive isomorphism of $M_{n}$ with $C_{n}$.

Proof. For $n=1$ the result is classical. Let $n>1$. Write $V^{n}=V^{n-1} \times V$ and $x=(z, y)\left(x \in V^{n}, z \in V^{n-1}, y \in V\right)$. Let $\chi \in C_{n}$. For each $z \in V^{n-1}$ there is a unique $c(z) \in V^{\prime}$ such that $\chi(z, y)=\psi(\langle c(z), y\rangle)$. Since $\psi\left(\left\langle c\left(z_{1}+\right.\right.\right.$ $\left.\left.\left.z_{2}\right)-c\left(z_{1}\right)-c\left(z_{2}\right), y\right\rangle\right)=1$ for all $y \in V$, we get $c\left(z_{1}+z_{2}\right)=c\left(z_{1}\right)+c\left(z_{2}\right)$. If $z_{r} \rightarrow z$ in $V^{n-1}$, then $\chi\left(z_{r}, y\right) \rightarrow \chi(z, y)$ uniformly on compacts in $y$, so that $c\left(z_{r}\right) \rightarrow c(z)$. Hence $c\left(V^{n-1} \longrightarrow V^{\prime}\right)$ is additive and continuous, hence 
$\mathbf{Q}_{p}$-linear. Write $c(z, y)=\langle c(z), y\rangle$. Then $c$ is linear in $z$ for fixed $y$ and linear in $y$ for fixed $z$, hence $n$-linear.

To show that the topologies are the same, notice first that on $M_{n}$ uniform convergence on compacts is the same as pointwise convergence since $M_{n}$ is finite dimensional. If $d_{r}$ are in $M_{n}$ and $\psi\left(d_{r}\right) \rightarrow 1$ uniformly on compacts of $V^{n}$ as $r \rightarrow \infty$, we must show that $d_{r} \rightarrow 0$ in $M_{n}$, i.e., $d_{r}\left(y_{1}, \ldots, y_{n}\right) \rightarrow 1$ for each fixed $\left(y_{i}\right) \in V^{n}$. Fixing $y_{1}, \ldots y_{n-1}$, we have $\psi\left(d_{r}\left(y_{1}, \ldots, y_{n-1}, y\right)\right) \rightarrow 1$ uniformly on compacts of $y$, and hence $d_{r}\left(y_{1}, \ldots, y_{n-1}, y\right) \rightarrow 0$ for each $y$.

Corollary 2. For any skew symmetric bilinear form $b$ on $V \times V, \psi(b)$ is a multiplier for $V$, and the map $b \mapsto[\psi(b)]$ induces an isomorphism of $\Lambda^{2}(V)$ with $H^{2}(V)$.

Proof. From [16] we know that the map that takes an alternating bicharacter $f$ to its class in $H^{2}(V)$ is an isomorphism. By the lemma above any $f$ is of the form $\psi(b)$ for a unique bilinear form on $V \times V$. Since $f$ is alternating, $\psi\left(b+b^{T}\right)=1$ where $b^{T}(x, y)=b(y, x)$. Hence $b+b^{T}=0$, showing that $b$ is skew symmetric. If $\psi(b)$ is the trivial multiplier, $\psi(b)$ is symmetric, hence, as before, $b=b^{T}$, hence $b=0$.

\section{Study of $H^{1}(G, U)$ for $p$-adic Lie groups $G$.}

We now study of $H^{1}(G, U)$ where $G$ is a $p$-adic Lie group and $U$ a finite dimensional vector space over $\mathbf{Q}_{p}$ on which $G$ acts linearly. We have seen that a map $f(G \longrightarrow U)$ is a 1-cocycle if and only if $\gamma_{f}: g \mapsto(f(g), g)$ is a morphism of topological groups from $G$ to $U \times^{\prime} G$. When $G$ is a $p$-adic Lie group, both groups are Lie and so $f$ becomes analytic [17]. So all cocycles are analytic and one can differentiate. Let $\mathfrak{g}$ be the Lie algebra of $G$ and $U$ that of $U$ so that $U \oplus^{\prime} \mathfrak{g}$ is the Lie algebra of $U \times^{\prime} G$. Its bracket is given by $\left[(u, Z),\left(u^{\prime}, Z^{\prime}\right)\right]=\left(Z \cdot u^{\prime}-Z^{\prime} \cdot u,\left[Z, Z^{\prime}\right]\right)$. Let $\partial \gamma_{f}$ be the differential of $\gamma_{f}$. Then $\partial \gamma_{f}$ is a Lie algebra morphism and is of the form $\partial \gamma_{f}(X)=(\partial f(X), X)$ for $X \in \mathfrak{g}\left(\partial f\right.$ defined by this equation). The relation $\partial \gamma_{f}([X, Y])=$ $\left[\partial \gamma_{f}(X), \partial \gamma_{f}(Y)\right]$ gives $\partial f([X, Y])=X \cdot \partial f(Y)-Y \cdot \partial f(X) \quad(X, Y \in \mathfrak{g})$ which defines a 1-cocycle for the Lie algebra $\mathfrak{g}$ with values in $U$. For any $X \in \mathfrak{g}$ let $\partial(X)$ be the left invariant vector field on $G$ differentiating by the rule $(\partial(X) g)(x)=(d / d t)_{t=0} g(x \exp t X)$ for $x \in G$ and analytic functions $g$ on open sets of $G$ containing $x$. Then

$$
(\partial f)(X)=(\partial(X) f)(1) .
$$


If we replace $f$ by $f+f^{\prime}$ where $f^{\prime}(g)=g \cdot u-u$ for some $u \in U$, then $\partial f^{\prime}(X)=\partial f(X)+X u$ showing that the cohomology class of $\partial f$ does not change. So $f \mapsto \partial f$ induces a map $\partial: H^{1}(G, U) \longrightarrow H^{1}(\mathfrak{g}, U)$. For any cocycle $f$ we write $[f]$ for its cohomology class. Notice that $H^{1}(G, U)$ is a $\mathbf{Q}_{p}$-vector space and $\partial$ is a $\mathbf{Q}_{p}$-linear map. Part (a) of the Theorem below is in [14]. $U^{G}$ is the space of vectors in $U$ fixed by $G$.

Theorem 2. (a) The kernel $\Gamma$ of the map $\partial\left(H^{1}(G, U) \longrightarrow H^{1}(\mathfrak{g}, U)\right.$ is the space of all cohomology classes which contain a cocycle vanishing on a compact open subgroup $K$ of $G$ (hence constant on the cosets $g K$ ).

(b) Suppose that for any $0 \neq u \in U$ the stabilizer $G_{u}$ of $u$ in $G$ is either all of $G$ or has measure zero in $G$. Then $\Gamma \simeq \operatorname{Hom}_{0}\left(G, U^{G}\right)$, the space of morphisms $G \longrightarrow U^{G}$ that vanish on compact open subgroups of $G$.

Proof. (a) We have $(\partial f)(X)=X u$ for some $u \in U$. If $f^{\prime}(g)=f(g)-(g \cdot u-u)$, then $f^{\prime} \simeq f$ and $\partial f^{\prime}=0$. We may thus assume $\partial f=0$. Since $\partial \gamma_{f}(X)=$ $(0, X)$ it is clear that $\gamma_{f}(g)=(0, g)$ for $g \in K$ where $K$ is a compact open subgroup of $G$. So $f(g)=0$ for $g \in K$. Hence $f(x h)=f(x)+x \cdot f(h)=f(x)$ for $x \in G, h \in K$. Conversely, if $f=0$ on $K, \partial f=0$.

(b) Let $f$ be a cocycle with $[f] \in \Gamma$. For fixed $x \in G$,

$$
f(h x)=f\left(x\left(x^{-1} h x\right)\right)=f(x) \quad\left(x^{-1} h x \in K \Leftrightarrow h \in x K x^{-1}\right) .
$$

For such $h$, we have $f(x)=f(h x)=f(h)+h \cdot f(x)$. We thus get $f(x)=$ $h \cdot f(x) \quad\left(h \in K_{x}:=K \cap x K x^{-1}\right)$. Suppose $f(x) \neq 0$. Then the subgroup $K_{x}$ which has measure $>0$ because it is open, is inside the stabilizer of $f(x)$ in $U$. Hence the stabilizer of $f(x)$ is all of $G$, i.e., $f(x) \in U^{G}$. The cocycle property implies that $f$ is a morphism vanishing on $K$, i.e., $f \in$ $\operatorname{Hom}_{0}\left(G, U^{G}\right)$. We claim that $f$ is the only cocycle in its class. If $f^{\prime}$ is another, then $f(x)-f^{\prime}(x)=x \cdot u-u$ for some $u \in U$. Thus $x \cdot u-u=0$ for $x$ in a compact open subgroup of $G$. As before we argue that $u \in U^{G}$, showing that $f=f^{\prime}$. Finally, it is clear that any $f \in \operatorname{Hom}_{0}\left(G, U^{G}\right)$ defines an element of $\Gamma$.

Lemma 4. If $G$ is compact, or more generally, generated by compact subgroups (=compactly generated), then $\operatorname{Hom}_{0}\left(G, \mathbf{Q}_{p}\right)=0$.

Proof. If $t$ is a homomorphism of $G$ into $\mathbf{Q}_{p}$ which is zero on a compact open subgroup $R$, and $K$ is any compact subgroup, $t$ is zero on $R \cap K$ which is 
open in $K$ and so the image $t(K)$ is a finite additive subgroup of $\mathbf{Q}_{p}$, hence 0 . So $t=0$ on $K$. Thus the kernel of $t$ contains all compact subgroups and so is all of $G$.

Lemma 5. Under the conditions of $(b)$ of Theorem 1, $\partial\left(H^{1}(G, U) \longrightarrow\right.$ $\left.H^{1}(\mathfrak{g}, U)\right)$ is injective if either $U^{G}=0$ or $\operatorname{Hom}_{0}\left(G, \mathbf{Q}_{p}\right)=0$, in particular if $G$ is compact.

Proof. Obvious, since $U^{G} \simeq \mathbf{Q}_{p}^{r}$.

Lemma 6. Suppose $G^{\sim} \longrightarrow G$ is a finite central cover of $G$ and we view $V$ (hence $V^{\prime}$ also) as a $G^{\sim}$-module. Then $H^{1}\left(G^{\sim}, V^{\prime}\right)=H^{1}\left(G, V^{\prime}\right)$.

Proof. Let $R$ be the kernel of $G^{\sim}$ to $G$. Then $R$ is central and for any cocycle $f\left(G^{\sim} \longrightarrow V^{\prime}\right)$ we have, with $h \in R, x \in G^{\sim}$,

$$
f(x h)=f(h x)=f(x)+x \cdot f(h)=f(h)+h \cdot f(x)=f(h)+f(x)
$$

since $R$ acts trivially on $V^{\prime}$. Thus $f(h) \in U^{G}$. So $\left.f\right|_{R}$ as a morphism of $R$ into $U^{G} \simeq \mathbf{Q}_{p}^{r}$ whose image is finite, as $R$ is finite. So the image is 0 , showing that $f$ is constant on the cosets of $R$. Hence $f$ descends to a cocycle on $G$, showing that $H^{1}\left(G, V^{\prime}\right) \longrightarrow H^{1}\left(G^{\sim}, V^{\prime}\right)$ is surjective. For injectivity, if the lift $f^{\sim}$ to $G^{\sim}$ of a cocycle $f$ on $G$ is trivial on $G^{\sim}, f(g)=f^{\sim}\left(g^{\sim}\right)=g^{\sim} \cdot u-u=g \cdot u-u$ for some $u \in V^{\prime}$, if $g$ is the image of $g^{\sim}$ in $G$.

\section{In which $G$ and $V$ are algebraic.}

Let $V$ be a finite dimensional vector space over $\mathbf{Q}_{p}$, and $G$ the group of $\mathbf{Q}_{p}$-rational points of a closed algebraic group $\mathbf{G} \subset \mathbf{G L}(\bar{V})$ defined over $\mathbf{Q}_{p}$, where $\bar{V}=\overline{\mathbf{Q}}_{p} \otimes_{\mathbf{Q}_{p}} V, \overline{\mathbf{Q}_{p}}$ being the algebraic closure of $\mathbf{Q}_{p}$. Then $G=\mathbf{G}\left(\mathbf{Q}_{p}\right)$ is a $p$-adic Lie group and the theory of the previous section applies. $V^{\prime}$ is the dual vector space to $V$.

Theorem 3. Suppose that $\mathbf{G}$ is connected semi simple, $G^{\sim} \longrightarrow G$ is a finite central cover of $G$, and we view $V$ (hence $V^{\prime}$ also) as a $G^{\sim}$-module. Then $H^{1}\left(G^{\sim}, V^{\prime}\right)=0$.

Proof. By lemma 6 it is enough to prove that $H^{1}\left(G, V^{\prime}\right)=0$. The key fact is that $H^{1}\left(\mathfrak{g}, V^{\prime}\right)=0$ since $\mathfrak{g}$ is semi simple. It is thus a question of verifying the conditions that make $\partial$ injective. For any $0 \neq u \in V^{\prime}$ the stabilizer of 
$u$ is the group of $\mathbf{Q}_{p}$-points of an algebraic subgroup $\mathbf{G}_{u} \subset \mathbf{G}$ defined over $\mathbf{Q}_{p}$ which is either a proper subvariety of the group $\mathbf{G}$ or all of $\mathbf{G}$. Coming down to $\mathbf{Q}_{p}$, we see that the stabilizer in $G$ is either all of $G$ or a proper subset given by the zeros of a finite number of polynomials with coefficients in $\mathbf{Q}_{p}$. Such a set has measure 0 in any $p$-adic manifold, a fact seen by using local coordinates. Thus, by Lemma 5, we need consider only the case when $V^{\prime G} \neq 0$. If $\mathbf{G}$ is $\mathbf{Q}_{p}$-anisotropic, $G$ is compact and we are done; if $\mathbf{G}$ is $\mathbf{Q}_{p}$-isotropic, then $G=(G, G)$, (see $[11]$ p. 200), and so $G$ has only trivial homoprohisms into $\mathbf{Q}_{p}$.

Remark 1. The map $H^{1}(G, U) \longrightarrow H^{1}(\mathfrak{g}, U)$ has no reason to be surjective because a Lie algebra morphism $\mathfrak{g} \longrightarrow U \oplus^{\prime} \mathfrak{g}$ lifts only to a morphism $K \longrightarrow U \times^{\prime} G$ of an open compact subgroup $K$ of $G$. Moreover one needs a group cocycle only on a small neighborhood of 1 in $G$ to get the Lie algebra cocycle. Thus only the inductive $\operatorname{limit}_{K} \lim ^{1}(K, U)$ where $K$ runs through the (diminishing) compact open subgroups of $G$ is associated to $H^{1}(\mathfrak{g}, U)$. For these questions, not only for $H^{1}$ but for all $H^{r}$ [18] [19]. Since we need only $H^{1}$ and our methods are elementary, based on [14], we have preferred to sketch our proofs.

\section{The Poincaré group over $\mathrm{Q}_{p}$.}

Let $V, G, \mathbf{G}$ be as in the previous section.

Theorem 4. Assume that $\mathbf{G}$ is semi simple, simply connected, with all of its simple factors defined and isotropic over $\mathbf{Q}_{p}$. Then $G^{\sim}$ exists, $G^{\sim} \longrightarrow G$ has finite kernel, and $\left(G^{\sim}, G^{\sim}\right)=G^{\sim}$. If $G$ acts irreducibly on $V$ and does not admit a non-zero skew symmetric invariant bilinear form on $V, P^{\sim}$ exists, $P^{\sim}=V \times^{\prime} G^{\sim}$, and $P^{\sim}=\left(P^{\sim}, P^{\sim}\right)$. In particular, all PUR's of $P^{\sim}$ are uniquely unitarizable by changing phase factors and all PUR's of $P$ can be uniquely unitarized after lifting to $P^{\sim}$. These results are valid if $V$ is $\mathbf{Q}_{p^{-}}$ isotropic, $G=\operatorname{Spin}(V)$ and $\operatorname{dim}(V \geq 3$ and $\neq 4$ with the proviso that in dimension 4, the Witt index of $V$ is maximal, i.e., equal to 2.

Proof. The general statements for $G$ follow from the works [10][11][12] when $\mathbf{G}$ is absolutely almost simple. For $\left(G^{\sim}, G^{\sim}\right)=G^{\sim}$ see [11], p. 268. The semi simple case follows easily. For $P$ we use Theorems 1 and 3, Proposition 1 , and Corollary 2. For $g \in G^{\sim}, v \in V$, the commutator $(g, v)=g \cdot v-v$ and 
these span $V$ as $V$ is irreducible. So $\left(P^{\sim}, P^{\sim}\right)$ contains $V$ and $G^{\sim}$, hence must be $P^{\sim}$.

Let us now treat the special case. Assume first that $\operatorname{dim}(V) \neq 4$. The action of $\operatorname{Spin}(V)$ on $V$ is irreducible and leaves a symmetric non-degenerate bilinear form invariant. Hence the only skew symmetric invariant bilinear form is 0 . The rest follows from the general case.

In dimension $4, V$ is hyperbolic and a model for it is the algebra $M$ of $2 \times 2$ matrices with det, the determinant, as the quadratic form. The group $\mathrm{SL}(2) \times \mathrm{SL}(2)$ acts on $M$ by $(a, b), X \longmapsto a X b^{-1}$ preserving the determinant, and so gives an isogeny of $\mathrm{SL}(2) \times \mathrm{SL}(2)$ into $\mathrm{SO}(M)$. So $\mathrm{SL}(2) \times \mathrm{SL}(2)=$ $\operatorname{Spin}(M)$. If $\mathbf{H}=\mathrm{SL}(2)$ and $H=\mathbf{H}\left(\mathbf{Q}_{p}\right)$, then $H^{\sim}$ exists as a finite cover over $H$. The result for $H \times H$ is immediate.

Remark 2. I cannot prove this if the Witt index is 0 or 1 in dimension 4.

Remark 3. The semi direct product structure of $P^{\sim}$ shows that the general pattern of particle classification does not change when we go from $P$ to $P^{\sim}$. My student Jukka Virtanen has studied the structure and symmetries of elementary particles over $\mathbf{Q}_{p}$ in his thesis [20]. Particles are still classified by mass and spin, except that the internal symmetry groups are subgroups of $\operatorname{Spin}(V)^{\sim}$ rather than of $\operatorname{Spin}(V)$, and so there are more of them. The particles of the $p$-adic world have richer internal structure.

\section{The Galilean group over $\mathbf{Q}_{p}$.}

Here spacetime $V=\mathbf{Q}_{p}^{r+1}$ has the decomposition into space and time: $V=$ $V_{0} \oplus V_{1}$ where $V_{0}=\mathbf{Q}_{p}^{r}, V_{1}=\mathbf{Q}_{p}$. The Galilean group is the semi direct product $G=V \times^{\prime} R$ where $R$ itself is the semi direct product of rotations and boosts. Thus $V_{0}$ is a $\mathbf{Q}_{p}$-isotropic quadratic vector space. We write $R_{0}$ for $\operatorname{Spin}\left(V_{0}\right)$ and set $R=V_{0} \times^{\prime} R_{0}$. We assume that $\operatorname{dim}\left(V_{0}\right) \geq 3, \neq 4$ with the proviso that in dimension 4 it is of maximal Witt index 2. The action of $G$ is defined by

$$
r=((u, \eta),(v, W)):(x, t) \longmapsto(W x+t v+u, t+\eta) .
$$

We write $(\cdot, \cdot)$ for the bilinear form on $V_{0}$. The dual $V^{\prime}$ consists of pairs $(\xi, t)$ with duality $\langle(\xi, t),(u, \eta)\rangle=(\xi, u)+t \eta$. The actions of the group $R_{0}$ on $V$ and $V^{\prime}$ are given by

$$
(v, W):(u, \eta) \longmapsto(W u+\eta v, \eta), \quad(v, W):(\xi, t) \longmapsto(W \xi, t-(W \xi, v)) .
$$


The Lie algebra actions are

$$
(v, W):(u, \eta) \longmapsto(W u+\eta v, 0), \quad(v, W):(\xi, t) \longmapsto(W \xi,-(\xi, v)) .
$$

As before the key is to compute $H^{1}\left(R, V^{\prime}\right)$ and $H^{1}\left(\mathfrak{r}, V^{\prime}\right)$ where $\mathfrak{r}=\operatorname{Lie}(R)$. We have $\mathfrak{r}=V_{0} \oplus \mathfrak{r}_{0}$ where $\mathfrak{r}_{0}=\operatorname{Lie}\left(R_{0}\right)$ and the sum is semi direct.

Let $\lambda\left(\mathfrak{r} \longrightarrow V^{\prime}\right)$ be a cocycle. Since $\mathfrak{r}_{0}$ is semi simple, we have $H^{1}\left(\mathfrak{r}_{0}, V^{\prime}\right)=$ 0 and so $\lambda(Z)=Z w$ for some $w \in V^{\prime}$ and all $Z \in \mathfrak{r}_{0}$. By subtracting from $\lambda$ the coboundary $X \longmapsto X w(X \in \mathfrak{r})$ we may assume that $\lambda$ itself vanishes on $\mathfrak{r}_{0}$. If we write $\lambda(v)=(L v, f(v))$, then $\lambda(v, Z)=(L v, f(v))$. Now the map $(\xi, t) \longmapsto \xi$ is an $\mathfrak{r}$-module morphism of $V^{\prime}$ onto $V_{0}^{\prime}$ (with action $(v, Z), \xi \longmapsto Z \xi)$, and $(v, Z) \longmapsto L v$ is a cocycle. The cocycle condition gives $L Z v=Z L v$ for all $v \in V_{0}, Z \in \mathfrak{r}_{0}$. Hence $L=c I$ for some constant $c$. The cocycle condition for $\lambda$ is $\lambda(Z v)=Z \lambda(v)$ for all $Z \in \mathfrak{r}_{0}, v \in V_{0}$. For $\lambda(v)=(c v, f(v))$ this becomes $(c Z v, f(Z v))=(c Z v, 0)$ so that $f(Z v)=0$. The $Z v$ span $V_{0}$ and so $f=0$. Hence $\lambda(v, Z)=c(v, 0)$. It is easy to verify that $\lambda_{0}:(v, Z) \longmapsto(v, 0)$ is a cocycle. It is not a coboundary; otherwise we will have, for some $\left(\xi_{0}, t_{0}\right),(v, Z) \cdot\left(\xi_{0}, t_{0}\right)=(v, 0)$ for all $v \in V_{0}, Z \in \mathfrak{r}_{0}$. Hence $\left(Z \xi_{0},-\left(\xi_{0}, v\right)\right)=(v, 0)$. This implies $\xi_{0}=0$ and hence $v=0$, a contradiction. Thus $H^{1}\left(\mathfrak{r}, V^{\prime}\right)$ is one dimensional with basis $\lambda_{0}$.

To determine $H^{1}\left(R, V^{\prime}\right)$ we shall first find a cocycle for $R$ which lifts $\lambda_{0}$. We search for it in the form $\theta:(v, W) \longmapsto(v, f(v, W))$. The cocycle condition gives

$$
f\left(v+W v^{\prime}, W W^{\prime}\right)=f(v, W)+f\left(v^{\prime}, W^{\prime}\right)-\left(W v^{\prime}, v\right) .
$$

Write $g(v)=f(v, I)$. Then, taking $W=W^{\prime}=I$ we get $g\left(v+v^{\prime}\right)=g(v)+$ $g\left(v^{\prime}\right)-\left(v^{\prime}, v\right)$ for which a solution is $g(v)=-(1 / 2)(v, v)$. Now $f\left(0, W W^{\prime}\right)=$ $f(0, W)+f\left(0, W^{\prime}\right)$ which implies $f(0, W)=0$. Hence

$$
f(v, W)=f((v, 1) \cdot(0, W))=f(v, 1)+(v, 1) \cdot f(0, W)=f(v, 1)=-\frac{1}{2}(v, v) .
$$

If we put $\theta(v, W)=(2 v,-(v, v))$ then it is a simple calculation that this defines a cocycle and that $\partial \theta=2 \lambda_{0}$. The cocycle $\theta$ is not a coboundary since $\partial \theta=2 \lambda_{0}$ is not, as we have already checked.

We claim that $H^{1}\left(R, V^{\prime}\right)$ is spanned by $\theta$. Now $\partial \theta$ spans $H^{1}\left(\mathfrak{r}, V^{\prime}\right)$, and the kernel of $\partial$ is 0 since it is $\simeq \operatorname{Hom}_{0}\left(R^{\sim}, \mathbf{Q}_{p}\right)$ by Theorem 2 as $V^{\prime R}$ has dimension 1. As $(R, R)=R$, we have $\operatorname{Hom}\left(R, \mathbf{Q}_{p}\right)=0$. 
For determining the multiplier we calculate first $\omega(a, g)=\psi\left(\theta\left(g^{-1}\right)(a)\right)$ (see $\S 3)$. Then

$$
\omega\left(\left(u^{\prime}, \eta^{\prime}\right),(v, W)\right)=\psi\left(-2\left(v, W u^{\prime}\right)-\eta^{\prime}(v, v)\right) .
$$

Write

$$
r=((u, \eta),(v, W)), \quad r^{\prime}=\left(\left(u^{\prime}, \eta^{\prime}\right),\left(v^{\prime}, W^{\prime}\right)\right) .
$$

From $\S 5$ we know then that a corresponding multiplier is

$$
m\left(r, r^{\prime}\right)=\psi\left(-2\left(v, W u^{\prime}\right)-\eta^{\prime}(v, v)\right) .
$$

Let

$$
m_{\tau}\left(r, r^{\prime}\right)=\psi\left(-2 \tau\left(v, W u^{\prime}\right)-\tau \eta^{\prime}(v, v)\right) \quad\left(\tau \in \mathbf{Q}_{p}\right) .
$$

Theorem 5. The $m_{\tau}$ are multipliers for the pseudo Galilean group $G=$ $V \times^{\prime} R$ and the map $\tau \longmapsto\left[m_{\tau}\right]$ is an isomorphism of $\mathbf{Q}_{p}$ with $H^{2}(G)$.

Remark 4. The form of the multiplier is superficially different from the one given in [7] (p. 287). But the two are equivalent. If $\nu\left(r, r^{\prime}\right)=\left(u, W v^{\prime}\right)-$ $\left(v, W u^{\prime}\right)+\eta^{\prime}\left(v, W v^{\prime}\right)$, then $\nu\left(r, r^{\prime}\right)-\mu\left(r, r^{\prime}\right)=f\left(r r^{\prime}\right)-f(r)-f\left(r^{\prime}\right)$ where $f(r)=f((u, \eta),(v, W))=(u, v)$.

Let

$$
\mu\left(r, r^{\prime}\right)=-2\left(v, W u^{\prime}\right)-\eta^{\prime}(v, v) .
$$

Then $\mu$ is a 2-cocycle with values in $\mathbf{Q}_{p}$ in view of the identity (verified in a routine manner)

$$
\mu(r, 1)=\mu(1, r)=0, \quad \mu\left(r_{1} r_{2}, r_{3}\right)+\mu\left(r_{1}, r_{2}\right)=\mu\left(r_{1}, r_{2} r_{3}\right)+\mu\left(r_{2}, r_{3}\right) .
$$

The multiplier $\mu$ allows us to build a t.c.e. $E$ of $G$ by $\mathbf{Q}_{p}$ with $E=G \times \mathbf{Q}_{p}$ and multiplication $(r, c)\left(r^{\prime}, c^{\prime}\right)=\left(r r^{\prime}, c+c^{\prime}+\mu\left(r, r^{\prime}\right)\right)$.

Theorem 6. We have $(E, E)=E$ and $E$ is the universal t.c.e. of $G$.

Proof. Since $(G, G)=G$, to prove that $(E, E)=E$ it is enough to show that $\{1\} \times \mathbf{Q}_{p} \subset(E, E)$. Take $r=((u, \eta),(v, 1)), \quad r^{\prime}=\left(\left(u^{\prime}, 0\right),(0,1)\right)$. Then $r$ and $r^{\prime}$ commute and $(r, c)\left(r^{\prime}, c^{\prime}\right)(r, c)^{-1}\left(r^{\prime}, c^{\prime}\right)^{-1}=(1, \zeta), \quad \zeta=$ $\mu\left(r, r^{\prime}\right)-\mu\left(r, r^{-1}\right)+\mu\left(r r^{\prime}, r^{-1}\right)$. Another calculation gives $\zeta=-2\left(v, u^{\prime}\right)$. Since $v$ and $u^{\prime}$ can be arbitrary it is clear that $\{1\} \times \mathbf{Q}_{p} \subset(E, E)$.

For the second assertion we note that $E$ covers each $E_{m_{\tau}}$ as a t.c.e. since $E \longrightarrow E_{m_{\tau}}$ given by $(r, c) \longmapsto(r, \psi(\tau c))$. Hence every PUR of $G$ lifts to a UR of $E$. Lemma 2 now shows that $E$ is the u.t.c.e. of $G$. 
Acknowledgment. I am deeply grateful to Professor Gopal Prasad for clarifying for me many aspects of the theory of groups over local fields. I would also like to thank Dr. Rahul Fernandez for his help with the preparation of this paper.

\section{References}

[1] E. G. Beltrametti and G. Cassinelli, G.,Quantum mechanics and p-adic numbers, 2 (1972), 1-7.

[2] E. G. Beltrametti, Can a finite geometry describe the physical spacetime? Universita degli studi di Perugia, Atti del convegno di geometria combinatoria e sue applicazioni, Perugia 1971, 57-62.

[3] E. G. Beltrametti Note on the p-adic generalization of Lorentz transformations, Discrete mathematics, 1(1971), 239-246.

[4] I. V. Volovich, Number theory as the ultimate theory, CERN preprint CERN-TH.4791/87, 1987.

[5] I. V. Volovich, p-adic string, Class. Quantum Grav. 4(1987) L83-L87.

[6] E. Wigner, On unitary representations of the inhomogeneous Lorentz group, Ann. Math., 40 (1939), 149-204.

[7] V. S. Varadarajan, Geometry of Quantum Theory, Springer Verlag, 2007.

[8] D. Wigner, Algebraic cohomology of topological groups, Trans. Amer. Math. Soc., 178(1973), 83-93.

[9] E. Michael, Selected selection theorems, Amer. Math. Monthly 63(1956), $233-238$.

[10] C. C. Moore, Group extensions of p-adic and adelic linear groups, Pub. Math. IHES, 35(1968), 5-70.

[11] G. Prasad and M. S. Raghunathan, Topological central extensions of semi simple groups over local fields, Ann. math., 119 (1984)(143-201; 203-268. 
[12] G. Prasad and A. S. Rapinchuk, Computation of the metaplectic kernel, Inst. Hautes tudes Sci. Publ. Math. No. 84 (1996), 91-187 (1997).

[13] G. W. Mackey, Unitary representations of group extensions. I, Acta Math., 99(1958), 265-311.

[14] J.-P. Serre, Sur les groupes de congruence des variétés abéliennes, Izv. Akad. Nauk. SSSR 28 (1964), 3-18; Collected Papers, Vol. II, pp. 230245 .

[15] J. A. Packer, Projective representations and the Mackey obstructionA survey, Group Representations, Ergodic Theory, and mathematical Physics: A Tribute to George W. Mackey., Cont. Math., 449., 345-378

[16] T. Digernes and V. S. Varadarajan, Models for the irreducible representation of a Heisenberg group, Inf. dim. anal., quantum prob. and related topics, 7(2004), 527-546.

[17] J.-P. Serre, Lie algebras and Lie groups, 1964 lectures given at Harvard University. Second edition. Lecture Notes in Mathematics, 1500. Springer-Verlag, Berlin, 1992.

[18] M. Lazard, Groupes analytiques p-adiques, Inst. Hautes tudes Sci. Publ. Math. No. 26 (1965).

[19] W. Casselman and D. Wigner, Continuous cohomology and a conjecture of Serre's, Inv. Math., 25(1974), 199-211.

[20] J. Virtanen, Thesis, 2008.

[21] C. C. Moore, Group extensions and cohomology for locally compact groups. IV Trans. Amer. Math. Soc., 221 (1976), 35-58. 